\title{
Correction to: Proactive Customized Protocol for Oral Management in Head and Neck Cancer Patients Planned for Radiotherapy
}

\author{
Deepika Kenkere $^{1}$ (D) Manjunath Narasappa Gudibande ${ }^{2}(D)$ C. Deepa $^{3}$ (D) \\ K. S. Srinath ${ }^{3}$ - K. R. Harshitha ${ }^{3}$ (D) Mallika P. Reddy ${ }^{3}$ (D
}

(C) The Association of Oral and Maxillofacial Surgeons of India 2021

Correction to: J. Maxillofac. Oral Surg.

$$
\text { https://doi.org/10.1007/s12663-021-01511-6 }
$$

In the original publication of the article, the article title was published incorrectly. The correct title is updated, and the original article has been corrected.

The original article can be found online at https://

doi.org/10.1007/s12663-021-01511-6.

Manjunath Narasappa Gudibande

dr.manjunathgn@gmail.com

Deepika Kenkere

deepikakenkere@gmail.com

C. Deepa

drdeepashekar@gmail.com

K. S. Srinath

drsrinath21@gmail.com

K. R. Harshitha

drharshitha.krishna@gmail.com

Mallika P. Reddy

mallikapreddy@gmail.com

1 Department of Dentistry, Fellow Oral Oncosurgery,

Department of ENT and Head \& Neck Surgery, Sri Devaraj

Urs Medical College, Tamaka, Kolar, India

2 Department of Radiotherapy, Sri Devaraj Urs Medical

College, Tamaka, Kolar, India

3 Department of Dentistry, Sri Devaraj Urs Medical College,

Tamaka, Kolar, India 